\title{
Avaliação da modulação autonômica da frequência cardíaca de pacientes com doença renal crônica em hemodiálise: estudo preliminar
}

Evaluation of the autonomic heart rate modulation of patients with chronic renal disease in bemodyalysis: preliminare study

Evaluación de la modulación autónoma de la frecuencia cardiaca en pacientes con enfermedad renal crónica sometidos a hemodiálisis: estudio preliminar

Natália Garbeto Rodrigues ${ }^{1}$, José Augusto Félix de Albuquerque ${ }^{2}$, Bruno Medeiros Guio ${ }^{3}$, Michel Silva Reis ${ }^{4}$

RESUMO I A doença renal crônica (DRC) é definida como dano à função renal. Doentes renais crônicos atingem alta prevalência de morte por eventos cardiovasculares antes dos estágios finais, sendo maior a mortalidade em estágio dialítico, em que é evidenciado um desequilíbrio autonômico. Objetivamos avaliar a modulação simpatovagal de pacientes com DRC em tratamento de hemodiálise ambulatorial. Foram avaliados 23 pacientes, divididos em: Grupo DRC com DRC no estágio 5D (DRC-5D) em tratamento regular de hemodiálise ambulatorial; Grupocontrole com indivíduos saudáveis. A variabilidade da frequência cardíaca (VFC) foi coletada por um cardiofrequencímetro e analisada por índices lineares do domínio do tempo e do domínio da frequência. 14 pacientes no Grupo DRC com média de idade 48ะ16; e 9 pacientes saudáveis no Grupo-controle com média de idade $64 \pm 5$. Nos resultados pelo domínio de tempo, - Grupo DRC mostrou valores significativamente maiores da frequência cardíaca (FC) comparado ao

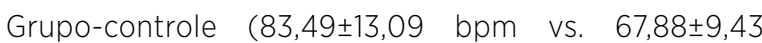
bpm). Todavia, os índices média dos intervalos R-R $(735,82 \pm 121,54$ ms vs. $898,94 \pm 123,58$ ms $)$, RMSSD $(11,75 \pm 11,86 \mathrm{~ms}$ vs. $20,03 \pm 6,80 \mathrm{~ms}), \operatorname{SDNN}(17,06 \pm 9,81 \mathrm{~ms}$ vs. $28,42 \pm 7,62 \mathrm{~ms}$ ) do Grupo DRC mostraram valores significativamente menores comparados aos do Grupo-controle, respectivamente. Nos resultados pelo domínio da frequência, o Grupo DRC mostrou valores significativamente menores em comparação ao Grupo-controle nos índices BFab $(129,7 \pm 184,3$ ms vs. $262,31 \pm 168,15 \mathrm{~ms})$ e AFab $(82,70 \pm 227,66 \mathrm{~ms}$ vs. $180,77 \pm 119,85$ ms). Pacientes com DRC em hemodiálise apresentaram redução da modulação parassimpática quando comparados com indivíduos saudáveis, sugerindo prejuízo do balanço simpatovagal e, consequente, disfunção autonômica cardíaca.

Descritores | Insuficiência Renal Crônica; Sistema Nervoso Autônomo; Doenças Cardiovasculares.

ABSTRACT I Chronic kidney disease (CKD) is defined as damage to kidney function. Patients have high prevalence of death by cardiovascular events before the final stages of the disease, with higher mortality in dialytic stage, in which autonomic imbalance is evidenced. This study seeks to evaluate sympathovagal modulation of patients with CKD undergoing hemodialysis treatment. We evaluated 23 subjects, divided into: CDK-group with CKD-stage 5D in regular hemodialysis treatment; and Control-group with healthy individuals. Heart rate variability (HRV) was collected by a heart rate monitor and analyzed by linear time domain and frequency domain indices. CDK-group had 14 patients, mean age of $48 \pm 16$; and Control-group had 9 healthy patients, mean age of $64 \pm 5$. In the time domain, CDK-group showed significantly higher heart

IUniversidade Federal do Rio de Janeiro - Rio de Janeiro (RJ) - Brasil. E-mail: msreis@hucff.ufrj. ORCID-0000-0002-6547-5803. 2Universidade Federal do Rio de Janeiro. - Rio de Janeiro (RJ) - Brasil. E-mail: msreis@hucff.ufrj. 3 Universidade Federal do Rio de Janeiro. - Rio de Janeiro (RJ) - Brasil. E-mail: msreis@hucff.ufrj. ORCID-0000-0003-4111-5739. ${ }_{4}^{4}$ Universidade Federal do Rio de Janeiro. - Rio de Janeiro (RJ) - Brasil. E-mail: msreis@hucff.ufrj.br. ORCID-0000-0002-3817-0529. 
rate values compared with Control-group $(83.49 \pm 13.09 \mathrm{bpm}$ vs. $67.88 \pm 9.43 \mathrm{bpm})$. However, the mean indices of the R-R intervals (735.82 $\pm 121.54 \mathrm{~ms}$ vs. 898.94 $\pm 123.58 \mathrm{~ms}$ ), RMSSD $(11.75 \pm 11.86 \mathrm{~ms}$ vs. $20.03 \pm 6.80 \mathrm{~ms})$, and SDNN $(17.06 \pm 9.81 \mathrm{~ms}$ vs. 28.42 $\pm 7.62 \mathrm{~ms}$ ) from CDK-group showed significantly lower values compared with Control-group. In frequency domain results, CDK-group showed significantly lower values compared with Control-group in LFab (129.7 \pm 184.3 ms vs. $262.31 \pm 168.15$ ms) and HFab ( $82.70 \pm 227.66$ ms vs. $180.77 \pm 119.85$ ms). Patients with CKD on hemodialysis had reduced parasympathetic modulation compared with healthy subjects, suggesting impairment of the sympathovagal balance and, consequently, cardiac autonomic dysfunction.

Keywords | Renal Insufficiency, Chronic; Autonomic Nervous System; Cardiovascular Diseases.

RESUMEN | La enfermedad renal crónica (ERC) se define como la pérdida de la función renal. Los pacientes renales crónicos tienen una alta prevalencia de muerte por eventos cardiovasculares antes de la etapa final, con mayor mortalidad en la etapa de diálisis, en la que se evidencia un desequilibrio autonómico. Este estudio tuvo como objetivo evaluar la modulación simpatovagal de pacientes con ERC sometidos a tratamiento de hemodiálisis ambulatoria. Se evaluaron 23 pacientes, lo cuales fueron divididos en: Grupo ERC con ERC en etapa 5D (ERC-5D) en tratamiento de hemodiálisis ambulatoria regular; Grupo de control con individuos sanos. Para identificar la variabilidad de la frecuencia cardíaca (VFC) se utilizó un monitor de frecuencia cardíaca y los datos obtenidos fueron analizados por los índices lineales de tiempo y de dominio de frecuencia. En el grupo ERC 14 pacientes tenían un promedio de edad de $48 \pm 16$ años; y en el grupo control 9 pacientes sanos tenían un promedio de edad de $64 \pm 5$. En los resultados para el dominio del tiempo, el grupo ERC mostró valores significativamente más altos de frecuencia cardíaca

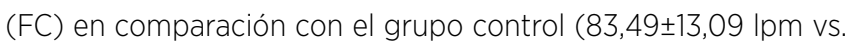
$67,88 \pm 9,43$ lpm). Sin embargo, los índices medios de los intervalos R-R (735,82 $\pm 121,54$ ms vs. 898,94 $\pm 123,58$ ms), RMSSD $(11,75 \pm 11,86$ ms vs. 20,03 $\pm 6,80$ ms), SDNN (17,06 $\pm 9,81$ ms vs. 28,42 $\pm 7,62$ ms) del grupo ERC demostraron valores significativamente más bajos en comparación con el grupo control, respectivamente. En los resultados para el dominio de la frecuencia, en el grupo ERC se obtuvo valores significativamente más bajos en comparación con el

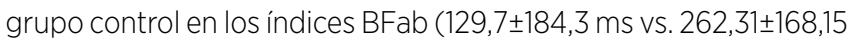

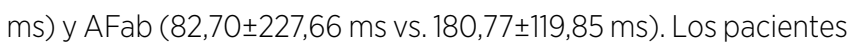
con ERC sometidos a hemodiálisis mostraron una modulación parasimpática reducida en comparación con los individuos sanos, lo que sugiere un deterioro del equilibrio simpaticovagal y, en consecuencia, una disfunción autonómica cardíaca.

Palabras clave | Insuficiencia Renal Crónica; Sistema Nervioso Autónomo; Enfermedades Cardiovasculares.

\section{INTRODUÇÃO}

A doença renal crônica (DRC) é considerada um problema de saúde pública mundial, com uma média de prevalência global de $13,4 \%{ }^{1}$. No Brasil, estima-se que há cerca de três a seis milhões de adultos com a doença ${ }^{2}$. A DRC é definida como perda gradual da função renal, independente de alteração estrutural do tecido e do diagnóstico causal, sendo estadeada com base no nível da taxa de filtração glomerular (TFG) ${ }^{3}$.

Pacientes renais crônicos são considerados indivíduos com alto risco para doenças cardiovasculares (DCV), pois possuem maior prevalência de seus fatores de risco considerados tradicionais, como idade avançada, hipertensão arterial sistêmica (HAS) e diabetes mellitus (DM), quando comparados à população geral. Além disso, a literatura também associa complicações cardiovasculares aos fatores de risco não tradicionais relacionados à DRC, como TFG diminuída e sobrecarga do volume extracelular ${ }^{4}$.
Sendo assim, estudos mostram que essa população atinge alta prevalência de mortalidade por eventos cardiovasculares antes mesmo dos estágios finais da doença, sendo maior a taxa de mortalidade em DRCs (estágio 5 - DRC-5D) em hemodiálise (HD) porque neste estágio é evidenciado um grande desequilíbrio da atividade autonômica ${ }^{2,5}$. Esse desequilíbrio na atividade simpatovagal influencia no controle do sistema cardiovascular, realizado parcialmente pelo Sistema Nervoso Autônomo (SNA), que é influenciado, dentre outros, por informações de barorreceptores, sistema respiratório, sistema vasomotor, sistema termorregulador e pelos rins, pois realizam a regulação hemodinâmica sistêmica através do sistema renina-angiotensinaaldosterona (SRAA), prostaglandinas e cininas renais ${ }^{6,7}$. Além disso, como dito anteriormente, indivíduos com DRC possuem outros fatores de risco (não tradicionais) que também influenciam na disfunção autonômica, como inflamação, estresse oxidativo e sobrecarga de volume extracelular. 
Este controle realizado pelo SNA está ligado intimamente à frequência cardíaca (FC), em que o aumento da FC é consequência de maior ação simpática e diminuição da parassimpática, isto é, inibição vagal; e a redução é consequência do predomínio da atividade parassimpática. A variabilidade da frequência cardíaca (VFC) é um método não invasivo capaz de avaliar as interações simpatovagais no controle central da FC, e indica a capacidade de resposta do coração a múltiplos estímulos ambientais e fisiológicos ${ }^{7}$.

A VFC é descrita como oscilações dos intervalos entre os batimentos consecutivos mostrados pelas ondas $\mathrm{R}$ do traçado eletrocardiográfico (iR-R). Alta VFC significa boa adaptação, característica de indivíduos com eficientes mecanismos autonômicos. Em contrapartida, baixa VFC é indicador de adaptação anormal e insuficiente do SNA, indicando a presença de mau funcionamento fisiológico no indivíduo. $\mathrm{Ou}$ seja, mudanças nos padrões da VFC podem fornecer um indicador antecipado e sensível de comprometimentos na saúde ${ }^{7}$.

A VFC pode ser avaliada por métodos lineares, mais comuns na prática clínica, pois estes podem ser obtidos em curtos e/ou em longos períodos de tempo. Dentre eles, estão os índices SDNN, SDANN e SDNNi, que traduzem a variabilidade global da FC e o componente de baixa frequência $(B F)$, que reflete predominantemente a modulação simpática. Já o rMMSD, pNN50 e componente de alta frequência (AF) representam a atividade parassimpática. Por fim, a relação $\mathrm{BF} / \mathrm{AF}$ representa o balanço simpatovagal sobre o coração ${ }^{7,8}$.

$\mathrm{Na}$ população com DRC, a redução da VFC está relacionada ao prejuízo do sistema parassimpático pelas alterações funcionais do SNA devido às toxinas urêmicas e pelo comprometimento estrutural das artérias ${ }^{5}$. Como consequência dessa disfunção, há uma maior incidência de hospitalização em pacientes DRC com baixa VFC, sugerindo que esse desequilíbrio autonômico possa participar da fisiopatologia da doença ${ }^{9}$. Adicionado a isso, autores demonstraram que a redução dos parâmetros SDNN, BF e BF/AF em pacientes em HD é um preditor independente de eventos adversos cardiovasculares, como arritmias, insuficiência cardíaca (IC) e morte súbita ${ }^{10-12}$.

Apesar das evidências iniciais indicarem que indivíduos com DRC possuem alteração da VFC, esta avaliação ainda não é rotineiramente empregada nesta população, diferente do que já ocorre em outras doenças crônicas (como doenças pulmonares obstrutivas crônicas, cardiopatias etc.). Diante do exposto, o objetivo do presente estudo foi avaliar a modulação simpatovagal de pacientes com DRC em HD. Neste sentido, justifica-se a concepção e desenvolvimento de mais pesquisas da modulação autonômica cardíaca de pacientes com DRC. Além disso, sua prática clínica poderia subsidiar a implementação de estratégias fisioterapêuticas, baseada em protocolos de exercício físico para reduzir o impacto dessa disfunção autonômica no risco cardiovascular.

\section{MÉTODOS}

\section{Participantes}

Trata-se de um estudo experimental, observacional, transversal e prospectivo. Para isso, os voluntários foram triados e estratificados em dois grupos: Grupo DRC, representado por pacientes com DRC em tratamento regular de HD ambulatorial no Hospital Universitário Clementino Fraga Filho (HUCFF) da Universidade Federal do Rio de Janeiro (UFRJ); e Grupo-controle, com indivíduos saudáveis sedentários. Os presentes estudos, assim como as coletas, foram desenvolvidos no Grupo de Pesquisa em Avaliação e Reabilitação Cardiorrespiratória (Gecare), do Departamento de Fisioterapia da UFRJ.

Os critérios de inclusão do grupo DRC foram: indivíduos acima de 18 anos, de ambos os sexos; sedentários e DRC-5D há pelo menos seis meses em tratamento ambulatorial, realizando hemodiálise (HD) três vezes por semana e quatro horas por sessão. Adicionalmente, o grupo controle foi composto por indivíduos acima de 18 anos, saudáveis de acordo com avaliação clínica, de ambos os sexos e sedentários (com prática de exercício físico menor que 150 minutos por semana). Foram excluídos do grupo DRC, os pacientes que tiveram internação hospitalar nos últimos três meses. Com relação ao grupo controle, foram excluídos os voluntários com histórico de doença cardiovascular, neurológica, ortopédica, muscular, metabólica e imunológica; e evidências clínicas e/ou funcionais de doença pulmonar obstrutiva crônica (razão volume expiratório forçado/capacidade vital forçada $\left.-\mathrm{VEF}_{1} / \mathrm{CVF}<70 \%\right)$.

Todos os voluntários assinaram o termo de consentimento livre e esclarecido e a privacidade dos 
sujeitos da pesquisa e confidencialidade dos dados foram totalmente garantidas durante todas as etapas do estudo.

\section{Protocolo experimental}

As coletas foram realizadas no setor de nefrologia, em sala climatizada, com temperatura entre $22^{\circ} \mathrm{C}$ e $24^{\circ} \mathrm{C}$, e todas ocorreram no mesmo período do dia (entre 5:30 e 6:00).

Os voluntários foram mantidos por 10 minutos em repouso na posição sentada, para que a $\mathrm{FC}$ atingisse valores basais. Após o repouso, foram coletados a FC e os iR-R, batimento a batimento, por 10 minutos na posição sentada, através de um cardiofrequencímetro (Polar ${ }^{\circledR}$ v800) com frequência de amostragem de 1000 $\mathrm{Hz}$, fixado por um cinto elástico no terço inferior do esterno e com transmissão simultânea para o relógio no qual foram armazenados. Posteriormente, os dados foram transportados e armazenados, por meio de uma interface USB, em microcomputador com software específico para que fossem analisados.

\section{Análise dos dados}

A VFC foi analisada por modelos matemáticos e estatísticos lineares no domínio do tempo e da frequência implementada por meio de rotina específica desenvolvida para este fim no aplicativo Kubius HRV 2.0 for Windows. Para que isso fosse possível, foi selecionado o trecho de maior estabilidade do sinal, com cinco minutos e livre de artefatos.

A análise no domínio do tempo foi realizada a partir dos índices: (1) média da FC e das variações da duração dos intervalos R-R (iR-R); (2) SDNN que se trata do desvio-padrão dos $\mathrm{iR}-\mathrm{R}$ normais em milissegundos(ms); e (3) rMSSD que corresponde à raiz quadrada da somatória do quadrado da diferença entre os iR-R consecutivos do registro do eletrocardiograma, dividido pelo número de $\mathrm{iR}-\mathrm{R}$ em um tempo determinado menos um, dos iR-R em $\mathrm{ms}^{7,8}$.
A análise no domínio da frequência foi realizada a partir das bandas de alta frequência (AF), bandas de baixa frequência (BF), ambas apresentadas em valores absolutos ( $\mathrm{ab})$. O AF reflete um predomínio de modulação vagal e o $\mathrm{BF}$ reflete um predominantemente a modulação simpática ${ }^{7,8}$.

\section{Análise estatística}

A análise estatística foi realizada no SigmaPlot for Windows versão 11.0, copyright(C 2008 Systat Software, Inc. Inicialmente, os dados foram submetidos ao teste de normalidade (teste de Shapiro-Wilk) e homogeneidade (teste de Levene). Na sequência, quando apropriado, foi aplicado teste T-Student não pareado ou teste de MannWhitney para as comparações dos índices no domínio do tempo e no domínio da frequência da VFC. Todas as medidas foram expressas em média e desvio-padrão com $\mathrm{p}<0,05$.

\section{RESULTADOS}

A população inicial deste estudo foi constituída de 24 pacientes elegíveis, e somente um paciente optou por não participar do estudo. Os participantes foram selecionados no período de dezembro de 2017 até fevereiro de 2018 nos Serviços de Nefrologia e no Departamento de Fisioterapia do Hospital Universitário Clementino Fraga Filho da UFRJ (Figura 1).

Neste sentido, todos completaram as avaliações, sendo 14 indivíduos no Grupo DRC, com média de idade de $48 \pm 16$ anos com 9 do sexo feminino; e 9 indivíduos no Grupo-controle, com média de idade $64 \pm 5$ anos sendo 5 do sexo feminino. No Grupo DRC, constituído por indivíduos com DRC-5D, as comorbidades associadas foram: HAS ( $n=9), \operatorname{DM}(n=5), I C(n=6)$, doença arterial coronariana $(n=5)$ e glomerulonefrite crônica $(n=2)$. Nenhum paciente era tabagista (tabela 1 ). 


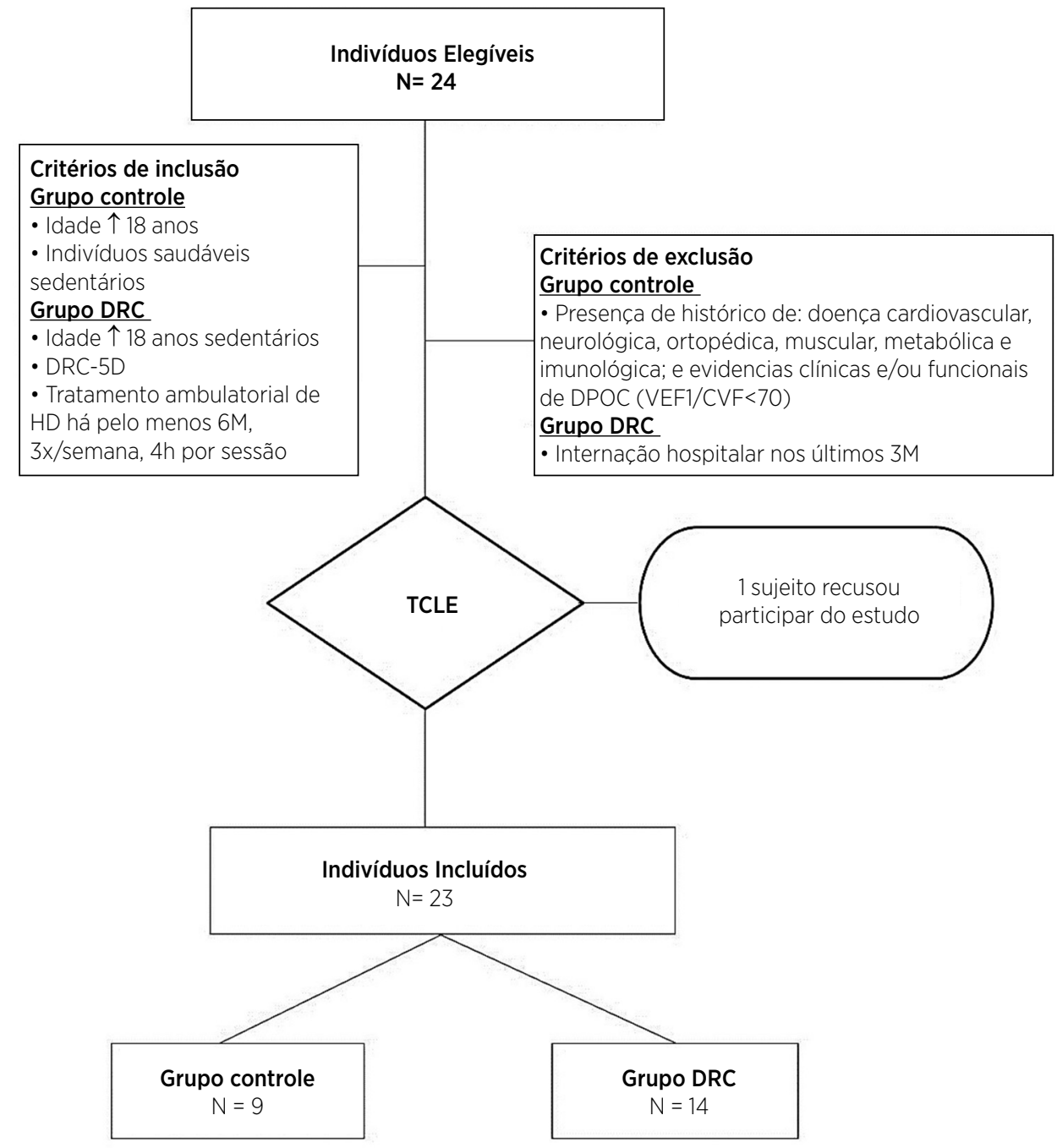

Figura 1. Fluxograma do estudo

DRC-5D: doentes renais crônicos em estágio 5; 6M: 6 meses; 3M: 3 meses; DPOC: doença pulmonar crônica obstrutiva; VEF1: volume expiratório forçado no 1ำ minuto; CVF: capacidade vital forçada; TCLE: termo de consentimento livre e esclarecido.

Tabela 1. Características gerais dos voluntários estudados

$\begin{array}{ccc} & \text { Grupo DRC } & \text { Grupo-controle } \\ \text { Pacientes analisados (n) } & 14 & 9 \\ \text { Idade (anos) } & 48 \pm 16 & 64 \pm 5 \\ \text { Sexo feminino - n (\%) } & 8(57,1) & 5(57,1) \\ \text { IMC }\left(\mathrm{kg} / \mathrm{m}^{2}\right) & 26 \pm 6 & 24 \pm 3 \\ \text { Betabloqueadores - n (\%) } & 3(21,4) & - \\ \text { Bloqueadores canal de cálcio- } \mathrm{n} & 4(28,5) & - \\ \text { (\%) } & 9(64,2) & - \\ \text { HAS - n (\%) } & 5(35,8) & - \\ \text { DM - n (\%) } & 6(42,5) & - \\ \text { IC - n (\%) } & 5(35,7) & - \\ \text { DAC - n (\%) } & 2(14,3) & -\end{array}$

IMC: índice de Massa Corporal; HAS: Hipertensão Arterial Sistêmica; DM: Diabetes Mellitus; IC: Insuficiência Cardíaca; DAC: Doença Arterial Coronariana.

$\mathrm{Na}$ Figura 2, nos gráficos (A, B, C e D) são apresentados os índices de domínio de tempo e da frequência através da $\mathrm{FC}, \mathrm{iR}-\mathrm{R}, \mathrm{SDNN}$ e BF. Foram observados maiores valores com significância estatística da média da FC no Grupo
DRC em comparação ao Grupo-controle $(83,49 \pm 13.09$ bpm vs. $67.88 \pm 9.43 \mathrm{bpm})(\mathrm{p}=0.006)$, e também mostraram piores ajustes autonômicos cardíacos e da VFC global do Grupo DRC em relação ao Grupo-controle, demostrado por valores significativamente menores na média dos $\mathrm{iR}-\mathrm{R}$ (735.82 $\pm 121.54 \mathrm{~ms}$ vs. $898.94 \pm 123.58 \mathrm{~ms})(\mathrm{p}=0.006)$, $\operatorname{SDNN}(17,06 \pm 9,81 \mathrm{~ms}$ vs. $28,42 \pm 7,62 \mathrm{~ms})(\mathrm{p}=0.005)$ e BFab (129.7 \pm 184.3 ms vs. $262,31 \pm 168,15 \mathrm{~ms})(\mathrm{p}=0,001)$, respectivamente.

Nos gráficos (E e F), também na Figura 2, são demonstrados os índices de domínio de tempo e frequência que representam predominância de atividade parassimpática (RMSSD e AFab). Foram observados valores significativamente menores no Grupo DRC em relação ao Grupo-controle nos seguintes parâmetros: $\operatorname{RMSSD}(11,75 \pm 11,86 \mathrm{~ms}$ vs. $20,03 \pm 6,80 \mathrm{~ms})$ ( $\mathrm{p}=0.003)$; e AFab $(82,70 \pm 227,66 \mathrm{~ms}$ vs. $180,77 \pm 119,85 \mathrm{~ms})$ $(\mathrm{p}=0.002)$, respectivamente. 
(A)

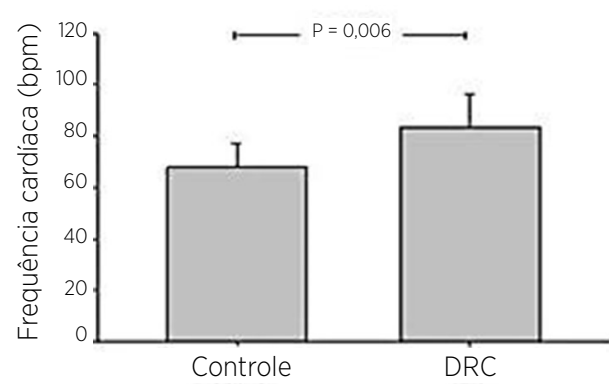

(C)

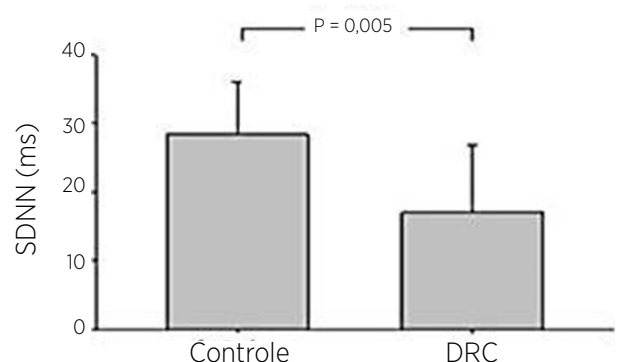

(E)

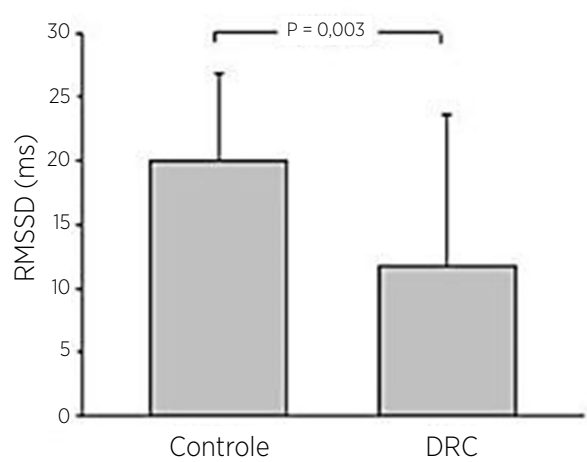

(B)

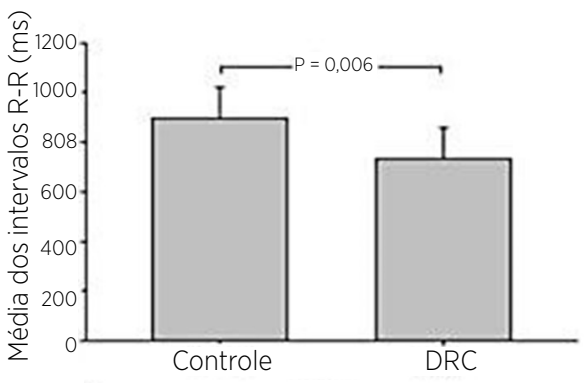

(D)

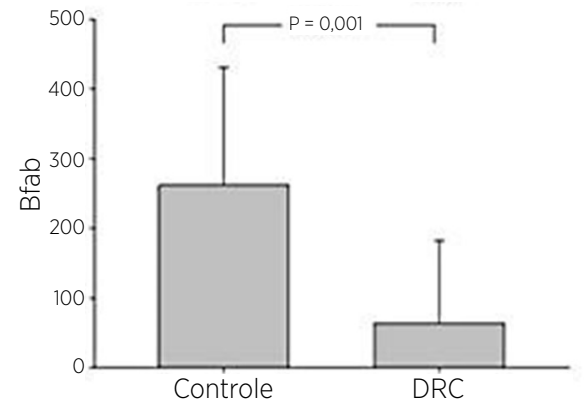

(F)

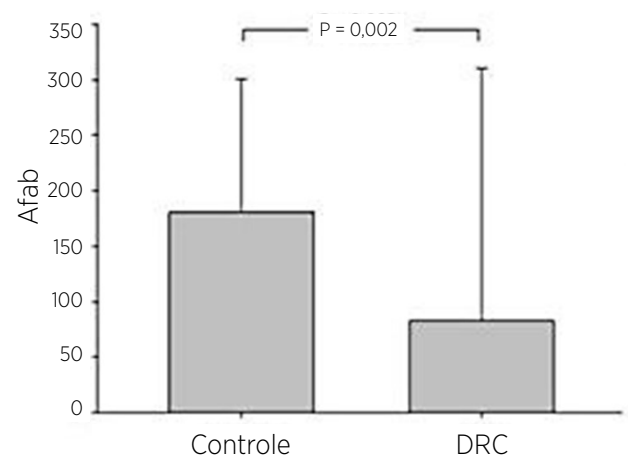

Figura 2. Resultados dos índices lineares globais (A, B, C e D) e parassimpáticos (E e F) da VFC no domínio de tempo e da frequência: (A) Frequência Cardíaca (bpm); (B) Média dos intervalos R-R (ms); (C) SDNN: desvio-padrão de todos os intervalos R-R (ms); (D) BFab: baixa frequência absoluta (ms); (E) rMSSD: raiz quadrada da média das diferenças entre os intervalos R-R (ms); ( $F$ ) AFab: alta frequência absoluta (ms).

\section{DISCUSSÃO}

Os resultados do presente estudo mostraram que indivíduos com DRC em HD possuem prejuízo na VFC com comprometimento da modulação vagal quando comparados aos indivíduos saudáveis pareados. Neste contexto, nefropatas crônicos em estágio terminal apresentam ajuste autonômico prejudicado e, consequentemente, pior variabilidade global através de menores valores nos índices iR-R, SDNN e BFab, assim como da média da FC.

Vita et al. ${ }^{13}$ corroboram com nossos achados, em que foi observado uma redução do BFab em comparação a indivíduos saudáveis, o que indica redução do balanço simpatovagal, isto é, baixa VFC. No mesmo sentido, o estudo de Chandra et al. ${ }^{14}$, envolveu 305 doentes renais crônicos de estágio moderado à terminal (estágios 3 a 5) e também observou, assim como no nosso estudo, que nefropatas crônicos em hemodiálise possuem menores valores de SDNN. Porém, eles observaram essa redução quando comparado à pacientes renais crônicos em estágio 3 da doença, sugerindo que ocorra uma piora progressiva da VFC global durante a evolução dos estágios da doença. Adicionado a isso, menores valores de $\mathrm{SDNN}$ em pacientes DRC estão relacionados à menores taxas de filtração glomerular ${ }^{9}$. Além desses achados, o mesmo estudo mostrou 
que, pacientes renais crônicos, desde o estágio 3,já possuem menores valores dos índices do domínio da frequência (AF, BF, BF/AF) e do SDNN em relação a indivíduos saudáveis ${ }^{13}$. Em consonância com nossos resultados, Thio et al. ${ }^{9}$ também estudaram os índices SDNN, rMSSD, AF, BF E BF/AF através do estudo PREVEND e encontraram que a prevalência de baixa VFC é significativamente maior em indivíduos com DRC.

De acordo com evidências disponíveis, a redução de tais índices prediz eventos adversos cardiovasculares, incluindo morte cardiovascular por todas as causas ${ }^{8,12,15,16}$. Inclusive, em um acompanhamento de 2,7 anos, os índices do domínio da frequência (AF, BF, BF/AF) foram os que mais predisseram desfechos nessa população, como eventos cardiovasculares (15\%), falência renal (22\%) e óbito (8\%) ${ }^{13}$.

Segundo autores, a VFC é mais suprimida em pacientes com DRC portadores de cardiopatia isquêmica, com doenças sistêmicas como diabetes mellitus ou amiloidose e naqueles com história de episódios hipotensores intradialíticos ${ }^{17-21}$. A VFC também pode ser afetada diretamente por fatores relacionados ao procedimento dialítico, como composição $0^{22}$ e temperatura do dialisato ${ }^{23}$, níveis de $\mathrm{PTH}^{24}$ e a duração da diálise de manutenção ${ }^{25}$.

$\mathrm{Na}$ modulação autonômica, tanto a retirada vagal quanto a hiperatividade simpática podem contribuir para a redução da $\mathrm{VFC}^{8}$. A patogenia da disfunção autonômica nessa população pode ser multifatorial, como pelo aumento da atividade simpática e/ou redução da atividade vagal devido à hiperatividade do SRAA e sinais aferentes liberados devido à isquemia renal que estimulam o fluxo simpático ${ }^{26-28}$. Nesta última condição, a ativação dos rins com aumento da reabsorção de sódio resulta em sobrecarga de fluidos, levando à exacerbação de doença renal e cardíaca ${ }^{29}$. Nesses pacientes com insuficiência renal combinada a doenças cardíacas, a baixa VFC também pode estar relacionada à presença de anormalidades estruturais do miocárdio ${ }^{30}$. Além disso, Salman ${ }^{31}$ ainda encontrou que o estresse mental também contribui para a diminuição da VFC nesses pacientes, já que este interfere nos fatores de risco da DRC, como alterações na atividade do SNA e do sistema neuroendócrino ${ }^{32}$.

Apontamos limitações neste estudo. O número pequeno de sujeitos de ambos os grupos deve ser considerado. No entanto, vale destacar à dificuldade de recrutar sujeitos idosos completamente saudáveis e também o quadro de gravidade de doentes renais crônicos, os quais apresentam alta frequência de intercorrências e internações.

Por fim, concluímos que pacientes com DRC em tratamento ambulatorial de hemodiálise apresentaram redução da modulação parassimpática quando comparados a indivíduos saudáveis, sugerindo prejuízo do balanço simpatovagal e, consequentemente, disfunção autonômica cardíaca.

\section{Aplicabilidade clínica}

Considerando a importância da atuação fisioterapêutica em pacientes com DRC em estágio dialítico, o presente estudo enxerga a avaliação por meio da VFC como uma ferramenta promissora, pois representa uma alternativa eficiente, segura e de baixo custo para a implementação de intervenções com objetivo de oferecer melhoria da saúde cardiovascular e qualidade de vida desses indivíduos.

\section{REFERÊNCIAS}

1. Hill NR, Fatoba ST, Oke JL, Hirst JA, O'Callaghan CA, Lasserson DS, et al. Global prevalence of chronic kidney disease - a systematic review and meta-analysis. PLoS One. 2016;11(7):e0158765. doi: 10.1371/journal.pone.0158765.

2. Marinho AWGB, Penha AP, Silva MT, Galvão TF. Prevalência de doença renal crônica em adultos no Brasil: revisão sistemática da literatura. Cad. Saude Colet. 2017;25(3):379-88. doi: 10.1590/1414-462X201700030134

3. Mayo Clinic Website [Internet]. Chronic Kidney Disease. Rochester (MN): Mayo Clinic; [cited 2020 Aug 4]. Available from: https://mayocl.in/3Aesl5A

4. Canziani MEF. Doenças cardiovasculares na doença renal crônica, J Bras Nefrol. 2004:26(3):20-1.

5. Reboredo MM, Pinheiro BV, Neder JA, Ávila MPW, Ribeiro MLBA, Mendonça AF, et al. Efeito do exercício aeróbico durante as sessões de hemodiálise na variabilidade da frequência cardíaca e na função ventricular esquerda em pacientes com doença renal crônica. J. Bras. Nefrol. 2010:32(4):372-79. doi:10.1590/ S0101-28002010000400006.

6. Aires MM. Fisiologia. 3rd ed. Rio de Janeiro: Guanabara Koogan; 2008

7. Vanderlei LCM, Pastre CM, Hoshi RA, Carvalho TD, Godoy MF. Noções básicas de variabilidade da frequência cardíaca e sua aplicabilidade clínica. Rev Bras Cir Cardiovasc. 2009;25(2):20517. doi: 10.1590/s0102-76382009000200018.

8. American Heart Association. Heart rate variability: Standards of measurement, physiological interpretation, and clinical use. Eur Hear J. 1996;17(3):354-81. doi: 10.1093/oxfordjournals. eurheartj.a014868.

9. Thio CHL, van Roon AM, Lefrandt JD, Gansevoort RT, Snieder $H$. Heart rate variability and its relation to chronic kidney disease. Psychosom Med. 2018;80(3):307-16. doi: 10.1097/ PSY.0000000000000556.

10. Cashion AK, Holmes SL, Arheart K, Acchiardo SR, Hathaway DK. Heart rate variability and mortality in patients with end stage renal disease. Nephrol Nurs J. 2005;32(2):173-84. 
11. Chandra P, Sands RL, Gillespie BW, Levin NW, Kotanko P, Kiser $M$, et al. Predictors of heart rate variability and its prognostic significance in chronic kidney disease. Nephrol Dial Transplant. 2012;27(2):700-709. doi: 10.1093/ndt/gfr340.

12. Fukuta H, Hayano J, Ishihara S, Sakata S, Mukai S, Ohte N, et al. Prognostic value of heart rate variability in patients with end-stage renal disease on chronic haemodialysis. Nephrol Dial Transplant. 2003;18(2):318-25. doi: 10.1093/ndt/18.2.318.

13. Vita G, Bellinghieri G, Trusso A, Costantino G, Santoro D, Monteleone F, et al. Uremic autonomic neuropathy studied by spectral analysis of heart rate. Kidney Int. 1999;56(1):232-237. doi: 10.1046/j.1523-1755.1999.00511.x.

14. Montano N, Ruscone TG, Porta A, Lombardi F, Pagani M, Malliani $A$, et al. Power spectrum analysis of heart rate variability to assess the changes in sympathovagal balance during graded orthostatic tilt. Circulation. 1994;90(4):1826-31. doi: 10.1161/01. CIR.90.4.1826.

15. Oikawa K, Ishihara R, Maeda T, Yamaguchi K, Koike A, Kawaguchi $\mathrm{H}$, et al. Prognostic value of heart rate variability in patients with renal failure on hemodialysis. Int J Cardiol. 2009;131(3):370-377. doi: 10.1016/j.ijcard.2007.10.033.

16. Rubinger D, Sapoznikov D, Pollak A, Popovtzer MM, Luria MH. Heart rate variability during chronic hemodialysis and after renal transplantation; studies in patients without and with systemic amyloidosis. J Am Soc Nephrol. 1999;10(9):1972-81. doi: 10.1681/ASN.V1091972.

17. Rubinger D, Backenroth R, Sapoznikov D. Restoration of baroreflex function in patients with end-stage renal disease after renal transplantation. Nephrol Dial Transplant. 2009;24(4):1305-13. doi: 10.1093/ndt/gfn732.

18. Giordano M, Manzella D, Paolisso G, Caliendo A, Varricchio M, Giordano $C$. Differences in heart rate variability parameters during the post-dialytic period in type II diabetic and nondiabetic ESRD patients. Nephrol Dial Transplant. 2001;16(3):566-73. doi: 10.1093/ndt/16.3.566.

19. Rubinger D, Revis N, Pollak A, Luria MH, Sapoznikov D. Predictors of hemodynamic instability and heart rate variability during hemodialysis. Nephrol Dial Transplant. 2004;19(8):2053-2060. doi: 10.1093/ndt/gfh306.

20. Barnas MGW, Boer WH, Koomans HA. Hemodynamic patterns and spectral analysis of heart rate variability during dialysis hypotension. J Am Soc Nephrol. 1999;10(12):2577-84. doi: 10.1681/ASN.V10122577.
21. Ferrario M, Raimann JG, Thijssen S, Signorini MS, Kruse A, DiazBuxo JA, et al. Effects of dialysate glucose concentration on heart rate variability in chronic hemodialysis patients: results of a prospective randomized trial. Kidney Blood Press Res. 2011;34(5):334-343. doi: 10.1159/000327851.

22. Zitt E, Neyer U, Meusburger E, Tiefenthaler M, Kotanko P, Mayer G, et al. Effect of dialysate temperature and diabetes on autonomic cardiovascular regulation during hemodialysis. Kidney Blood Press Res. 2008;31(4):217-225. doi: 10.1159/000141926.

23. Polak G, Stroyecki P, Grzesk G, Manitius J, Grabczewska Z, Przybyl R. Effect of parathormone on heart rate variability in hemodialysis patients. Auton Neurosci. 2004;115(1-2):94-98. doi: 10.1016/j.autneu.2004.08.002

24. Mylonopoulou M, Tentolouris N, Antonopoulos S, Mikros S, Katsaros K, Melidonis A, et al. Heart rate variability in advanced chronic kidney disease with or without diabetes: midterm effects of the initiation of chronic hemodialysis therapy. Nephrol Dial Transplant. 2010;25(11):3749-3754. doi: 10.1093/ndt/gfq226.

25. Herzog CA, Mangrum JM, Passman R. Sudden cardiac death and dialysis patients. Semin Dial. 2008;21(4):300-307. doi: 10.1111/j.1525-139X.2008.00455.x.

26. Vonend O, Rump LC, Ritz E. Sympathetic overactivity - the Cinderella of cardiovascular risk factors in dialysis patients. Semin Dial. 2008;21(4):326-330. doi: 10.1111/j.1525-139X.2008.00456.x.

27. Koomans HA, Blankestijn PJ, Joles JA. Sympathetic hyperactivity in chronic renal failure: a wake-up call. J Am Soc Nephrol. 2004;15(3):524-537. doi: 10.1097/01.asn.0000113320.57127.b9.

28. McDonough AA. Mechanisms of proximal tubule sodium transport regulation that link extracellular fluid volume and blood pressure. Am J Physiol Regul Integr Comp Physiol. 2010;298(4):R831-R861. doi: 10.1152/ajpregu.00002.2010.

29. Leineweber K, Heinroth-Hoffmann I, Pönicke K, Abraham G, Osten B, Brodde OE. Cardiac beta-adrenoreceptors desensitization due to increased beta-adrenoreceptor kinase activity in chronic uremia. J Am Soc Nephrol. 2002;13(1):117-24. doi: 10.1681/ASN.V131117.

30. Salman IM. Cardiovascular autonomic dysfunction in chronic kidney disease: a comprehensive review. Curr Hypertens Rep. 2015;17(8):1-20. doi: 10.1007/s11906-015-0571-z.

31. Bruce MA, Beech BM, Sims M, Brown TN, Wyatt SB, Taylor HA, et al. Social environmental stressors, psychological factors, and kidney disease. J Investig Med. 2009;57(4):583-89. doi: 10.2310/JIM.0b013e31819dbb91. 